\title{
溶剂极性对D-A-D型延迟苂光分子电荷转移激 发态的调控
}

\author{
何桂营 ${ }^{1,2}$, 匡卓然 1,2 , 王娴 1,2 , 宋宏伟 1,2 , 郭前进 $1^{*}$, 夏安东 ${ }^{1,2}$ \\ 1. 中国科学院化学研究所, 光化学院重点实验室, 北京分子科学国家实验室, 北京 100190 \\ 2. 中国科学院大学, 北京 100049 \\ *通讯作者, E-mail: guoqj@iccas.ac.cn; andong@iccas.ac.cn
}

收稿日期: 2017-09-13; 接受日期: 2017-10-09; 网络版发表日期: 2017-11-20

国家自然科学基金(编号: 21333012, 21373232)和国家重点基础研究发展计划(编号: 2013CB834604)资助项目

\begin{abstract}
摘要恶二唑基四极分子(2,5-bis(4-(10H-phenoxazin-10-yl)phenyl-1,3,4-oxadiazole, 2PXZ-OXD)具有典型的 电子给体(donor)-受体(acceptor)-给体(donor) (D-A-D)的结构特点, 表现出优良的延迟荧光特性. 我们利用稳 态、瞬态光谱方法研究了该分子在不同极性溶剂中的光物理特性,发现随着溶剂极性的增加, Stokes位移明 显增加, 证明了光激发下 2 PXZ-OXD分子产生了强偶极性的电荷转移态。进一步的量化计算结果发现, 单重 激发态的偶极矩显著大于三重激发态的偶极矩, 溶剂极性对单重激发态的影响更大. 溶剂极性的增加, 打破 了四极分子的对称性, 促进了单重态能级的下降, 从而调控单-三重态间能级差, 导致延迟荧光特性的改变. 溶剂极性在对单重态与三重态的能级差调控的同时, 也会对2PXZ-OXD分子的发射振子强度有影响, 强极性 造成的显著非辐射驰豫过程会明显降低发光性能.
\end{abstract}

关键词电荷转移, 延迟荧光, 溶剂化

\section{1 引言}

有机发光二极管 (organic light-emitting diode, OLED)由于具有超薄、重量轻、功耗低、柔性等特 点, 在新一代的显示技术中受到了广泛的关注 ${ }^{[1]}$. 对于 利用单重态发光的苂光材料, 发光器件的量子效率理 论上低于 $25 \%$, 为了提高器件的效率, 三重态的利用成 为OLED发光材料研究的热点. 包含稀有金属的磷光 材料可以有效地将单重态和三重态激子转变为光子. 一般情况下, 由于强的自旋轨道耦合可以有效诱导系 间窝越并产生三重态激子, 重原子效应可以用来发展
一系列的磷光材料. 因此, 含铱、锇和铂等的过渡金 属配合物用作OLED中高效的光致磷光掺杂材料 $[2,3]$.

而对基于有机小分子的OLED材料来说, 通过最小 化单重态和三重态激子之间的能隙 $\left(\Delta E_{\mathrm{ST}}\right)$, 可以通过 热活化实现三重态到单重态的反向系间窖越(reverse intersystem crossing, RISC), 理论上可以把所有的电致 激发态都转化为光, 这种材料表现出来的远大于单重 态激子寿命的苂光寿命的现象称为热活化的延迟苂光 (thermally activated delayed fluorescence, TADF). 设计具 有很小的单-三重态间能隙的小分子, 可能通过控制最 高占据轨道(highest occupied molecular orbital, HOMO)

引用格式: 何桂营, 匡卓然, 王涃, 宋宏伟, 郭前进, 夏安东. 溶剂极性对D-A-D型延迟苂光分子电荷转移激发态的调控. 中国科学: 化学, 2017, 47: He G, Kuang Z, Wang X, Song H, Guo Q, Xia A. Solvent polarity dependence on excited charge-transfer state properties in donor-acceptor-donor thermally activated delayed-fluorescence molecule. Sci Sin Chim, 2018, 48: 210-217, doi: 10.1360/N032017-00149 
和最低未占据轨道(lowest unoccupied molecular orbital, LUMO)之间的空间重叠来实现. 在电子给受体(D-A) 的分子中, HOMO轨道和LUMO轨道通常会分别占据 电子给体和电子受体部分, 从而减小两者的轨道重叠. 有文献报道, 一些电子给受体的分子对三重态的捕获 效率已经接近 $100 \%{ }^{[4-6]}$.

尽管在器件效率的提高上已取得了一定的进展, 但是分子结构与能级对其影响的方式始终不清楚. 具 有强偶极激发态的分子的能级会受到环境的影响, 包 括溶剂及聚合物基底的极性等. 环境诱导的激发态 分子结构重组会进一步降低发光态能级导致发射峰 红移 ${ }^{[712]}$. 实验与理论结果证明, 相比较D-A型体系, D-A-D型的四极矩分子表现出更为有效的延迟苂光与 发光效率 ${ }^{[13,14]}$. 本文选择D-A-D型分子 $(2,5-b i s(4-(10 H-$ phenoxazin-10-yl)phenyl-1,3,4-oxadiazole, 2PXZ-OXD), 对其电荷转移特征及激发态动力学进行了研究, 阐释 了溶剂极性环境对 $2 \mathrm{PXZ}-\mathrm{OXD}$ 分子能级调控及结构弛 豫的影响.

\section{2 实验部分}

\section{1 试剂与仪器}

实验用的恶二唑基分子(2PXZ-OXD)购于机光科技 股份有限公司(Luminescence Technology Corp., 中国), 合成及表征方法见文献[14]. 图1是2PXZ-OXD分子结 构图. 实验中用到的所有溶剂, 包括环己烷 $(\mathrm{CHX})$ 、甲 苯 $(\mathrm{TOL})$ 、三氯甲烷 $(\mathrm{TCM})$ 、四氢呋喃 $(\mathrm{THF})$ 、二氯甲 烷(DCM) 和乙腈(ACN), 纯度为分析纯. 苂光量子产率 标准物二苯基葱(DPA)购于百灵威科技公司, 其量子 产率为 0.95 (环己烷溶液).

稳态紫外-可见吸收及苂光光谱分别使用日本日 立公司的U-3010吸收光谱仪和F-4600苂光光谱仪测 量. 飞秒瞬态吸收实验则使用本课题组自行搭建的飞 秒瞬态吸收光谱仪完成. 纳秒瞬态吸收实验使用商用 仪器LP920, 同时使用其发光模式对延迟苂光进行测 量. 快速苂光寿命使用英国爱丁堡公司的F900时间相 关单光子计数 (TCSPC) 光谱仪测量.

\section{2 实验方法}

\subsection{1 量子化学计算}

2PXZ-OXD的基态 $\left(\mathrm{S}_{0}\right)$ 和最低激发态的三重态 $\left(\mathrm{T}_{1}\right)$

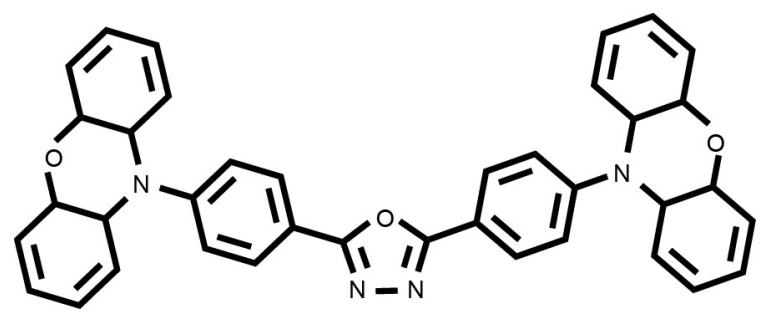

图 1 恶二唑基2PXZ-OXD分子结构图

构型都是基于密度泛函(DFT)的方法, 特别的对于最 低激发态的单重态 $\left(\mathrm{S}_{1}\right)$ 使用含时密度泛函(TDDFT), 计 算选用M062X泛函, 基组为6-31 $\mathrm{g}^{*}$. 所有构型优化及能 量计算都是在气相条件下进行的. M062X泛函更适用 于具有电荷转移特性的 2 PXZ-OXD的激发态计算 ${ }^{[15]}$. 基态、激发态的特性利用自然跃迁轨道方法(NTO)进 行分析 ${ }^{[16]}$. 所有的计算都是基于 Gaussian 09 软件包 ${ }^{[17]}$.

\subsection{2 苂光寿命测量}

快速荧光寿命使用 TCSPC光谱仪完成, 激发波 长选择为 $370 \mathrm{~nm}$. 延迟苂光测量使用闪光光解装置 (LP920, Edinburgh Instruments, UK) 的发光模式完成, 激发波长选择为 $355 \mathrm{~nm}$, 并在 $\mathrm{N}_{2}$ 饱和条件下测量.

\subsection{3 瞬态吸收光谱}

飞秒时间分辨的瞬态吸收光谱使用自行搭建的 宽带原浦-探测(pump-probe)装置 ${ }^{[10,18,19]}$. 钣蓝宝石飞秒 激光器 (Coherent, Legend Elite, USA)产生 $800 \mathrm{~nm}$ 的基 频光, 从放大级激光器得到的脉冲用 $90 / 10$ 分束镜分成 一束较强的原浦光和一束较弱的探测光. 强原浦光通 过 BBO 晶体倍频产生 $(400 \mathrm{~nm})$ 用于激发样品. 另一束 较弱的光通过光学延迟后聚焦在厚度为 $2 \mathrm{~mm}$ 的水中 产生宽带的超连续白光 $(425 \sim 780 \mathrm{~nm})$, 利用 $50 / 50$ 分束 镜分别产生信号光与参考光. 每次测量中都对有葲浦 光的信号光和参考光 $\left(I_{\mathrm{probe}}^{\mathrm{on}}(t, \lambda)\right.$ 和 $\left.I_{\mathrm{ref}}^{\mathrm{on}}(t, \lambda)\right)$ 和没有㫤浦 光时的 $\left(I_{\mathrm{probe}}^{\text {off }}(t, \lambda)\right.$ 和 $\left.I_{\text {ref }}^{\text {off }}(t, \lambda)\right)$ 进行测量, 瞬态吸收的差 谱可以通过以下公式计算得到:

$$
\Delta A(t, \lambda)=-\log \left(\frac{I_{\text {probe }}^{\text {on }}(t, \lambda)}{I_{\text {probe }}^{\text {off }}(t, \lambda)} \times \frac{I_{\text {ref }}^{\text {off }}(t, \lambda)}{I_{\text {ref }}^{\text {on }}(t, \lambda)}\right)
$$

样品的浓度调整为 $1 \mathrm{~mm}$ 光程样品池中的吸光度 为 0.3 . 各向同性的瞬态吸收光谱通过半波片调节泵浦 光和探测光的偏振夹角为 $54.7^{\circ}$ 来获得.

飞秒瞬态吸收数据分析过程中, 原始数据首先要 
经过白光色散校正. 校正后数据通过基于 TIMP的全 局拟合软件Glotaran完成. 使用目标模型对数据拟合 得到组分相关光谱(SAS)及相应的浓度衰减 ${ }^{[2,21]}$.

纳秒瞬态吸收光谱使用LP920闪光光解装置完成. 每次测试中, $\mathrm{N}_{2}$ 饱和样品时鼓气 $10 \mathrm{~min}$. 控制样品吸光 度在激发波长处约 $0.3 \mathrm{OD}$.

\section{3 结果与讨论}

\section{1 稳态吸收与苂光光谱}

图2所示为2PXZ-OXD在不同极性溶剂中的归一 化的吸收光谱和苂光光谱. 并在表 1 中列出了不同溶 剂极性相关的光谱参数. 位于 $400 \mathrm{~nm}$ 附近的低能量吸 收带归属于分子内电荷转移(ICT)态的吸收. 尤其值得 注意的是, 这一分子的吸收峰在极性溶剂中稍微蓝移, 这可能是基态分子与溶剂相互作用, 使得在极性溶剂 中基态分子能量下降, 并且极性越大, 能量下降越明 显. 例如, 在强极性的乙腈溶液中相对于非极性溶剂 环已烷, 蓝移了 $15 \mathrm{~nm}^{[9]}$.

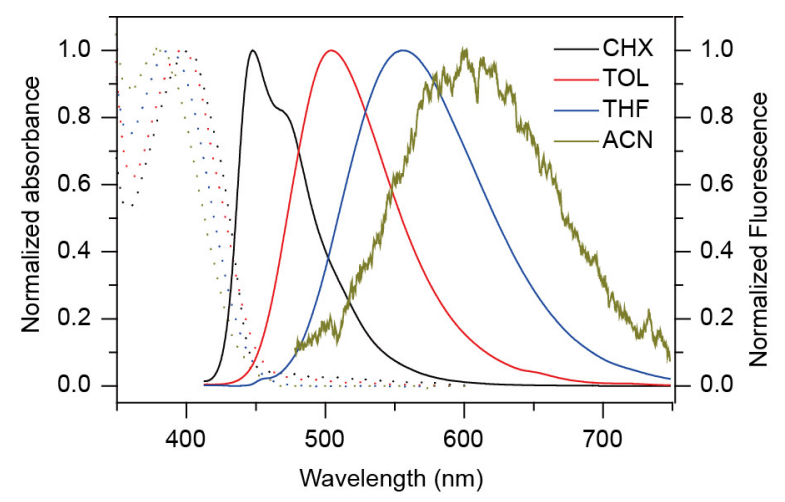

图 2 不同溶剂中的归一化吸收(虚线)与荧光光谱(实线) (网络版彩图).
而与吸收光谱蓝移特征不同的是, 2PXZ-OXD的 荧光光谱随着溶剂极性的增加而产生的是明显红移 现象, 这是具有分子内电荷转移态的溶剂化作用的典 型特征. 从环己烷到乙腈, 苂光峰红移了近 $150 \mathrm{~nm}$, 并 且随着溶剂极性的增加, 荧光峰的光谱宽度从 $60 \mathrm{~nm}$ 增加到了 $130 \mathrm{~nm}$ 左右. 尤其是只有在非极性的环己烷 中才能观测到荧光峰的振动特点. 由于在非极性溶剂 中的发光带与吸收带呈镜像关系, 发光可能来自于同 一个激发态. 而随着溶剂极性的增加, 激发态分子构 型的变化导致了溶剂化诱导的光谱红移和光谱展宽. 这就意味着激发态分子内电荷转移形成了极性更大 的发光态 ${ }^{[10,22]}$.

\section{2 溶剂化效应}

为了进一步理解溶剂化效应及 $2 \mathrm{PXZ}-\mathrm{OXD}$ 分子的 激发态强极性特点, 我们分析了不同溶剂中的Stokes 位移与溶剂极性之间的相互关系. 如图2所示, 随着 溶剂极性的增加, 2PXZ-OXD的吸收光谱具有轻微蓝 移, 而荧光光谱却具有更加显著的红移. 这足以证 明在强极性溶剂中, 2PXZ-OXD的激发态是从最初的 Franck-Condon态驰豫到一个极性更大的电荷转移态, 从而导致更大的Stokes位移. Stokes位移与溶剂极性参 数可以通过以下的Lippert-Mataga方程给出 ${ }^{[23,24]}$

$$
\Delta v=v_{\mathrm{abs}}-v_{\mathrm{flu}}=\frac{2 \Delta \mu^{2}}{h c a^{3}} \Delta f+\text { const. }
$$

其中, $\Delta v$ 是在不同溶剂中的Stokes位移, $v_{\mathrm{abb}}$ 和 $v_{\mathrm{flu}}$ 分别 是吸收峰与发射峰的波数, $h$ 是Planck常数, $c$ 是光速, $a$ 是Onsager半径, $\Delta \mu\left(\Delta \mu=\mu_{e}-\mu_{g}\right)$ 则是激发态与基态 偶极矩的差, $\Delta f$ 是溶剂极性参数, 可以通过溶剂的介 电常数 $\varepsilon$ 和折光度 $n$ 得到:

表 1 不同溶剂的极性参数与2PXZ-OXD在相应溶剂中的光谱性质

\begin{tabular}{ccccccc}
\hline & $\Delta f$ & $\lambda_{\text {abs }}(\mathrm{nm})$ & $\lambda_{\text {flu }}(\mathrm{nm})$ & ${\text { Stokes shift }\left(\mathrm{cm}^{-1}\right)}^{\text {Lifetime }(\mathrm{ns})^{\text {a) }}}$ & Quantum yield $^{\mathrm{b})}$ \\
\hline CHX & -0.003 & 402 & 450 & 2653 & 1.79 & 0.09 \\
TOL & 0.0159 & 398 & 504 & 5284 & 8.51 & 0.18 \\
TCM & 0.149 & 399 & 540 & 6544 & 10.9 & 0.13 \\
THF & 0.210 & 387 & 556 & 7854 & 8.26 & 0.07 \\
DCM & 0.218 & 391 & 565 & 7876 & 9.72 & 0.06 \\
ACN & 0.305 & 385 & 605 & 9445 & 0.47 & 0.006 \\
\hline
\end{tabular}

a) 快速苂光寿命使用TCSPC测得, 激发波长为 $370 \mathrm{~nm}$, 仪器响应函数约为 $350 \mathrm{ps}$; b) 苂光量子产率使用二苯基葱环已烷溶液( 0.95$)$ 作 为标准物 


$$
\Delta f=\frac{\varepsilon-1}{2 \varepsilon+1}-\frac{n^{2}-1}{2 n^{2}+1}
$$

图3给出了2PXZ-OXD分子的Stokes位移和溶剂极 性的Lippert-Mataga关系图. 相应的参数列于表 1 . 为 了进一步定量确定分子内电子转移激发态的极性, 我 们通过Lippert-Mataga关系式得到激发态与基态偶极 矩差 $\Delta \mu . \Delta \mu$ 直接反映了激发后, 激发态分子的偶极矩 相对于基态时的偶极矩的变化. 通过在同一级别上的 DFT计算, 得到Onsager半径约为 $6.47 \AA^{[25]}$, 从而估算出 这一分子的激发态与基态偶极矩差 $\Delta \mu$ 约为 $24.5 \mathrm{D}$. 如 此之大的偶极矩差进一步证明了2PXZ-OXD具有显著 的分子内电子转移, 与下面的量化计算的结果一致.

\section{3 量子化学计算}

通过对 2PXZ-OXD分子的稳态吸收与苂光光谱及 其溶剂化的研究, 可以得到这一分子具有明显的激发 态电荷转移特性. 通过量子化学计算, 可以进一步证明 这一特点. 构型优化的结果显示, D-A-D型2PXZ-OXD 分子的基态结构保持对称性, 而激发态分子的结构对 称性被打破, 形成了强偶极的电荷转移态 ${ }^{[26,27]}$. 如图 4所 示为分别基于基态和激发态稳定构型的自然跃迁轨 道. 基于自然跃迁轨道分析, 空穴(hole)轨道定域在两 端的吩恶嗪部分, 而电子(electron)轨道则定域在中心 基团二苯恶二唑单元, 证明了吩恶嗪的给电子和恶二 唑的吸电子特征.

基态稳定结构下的NTO分析可以看出, 从两侧的 电子给体到中心电子受体, 2PXZ-OXD具有明显的对

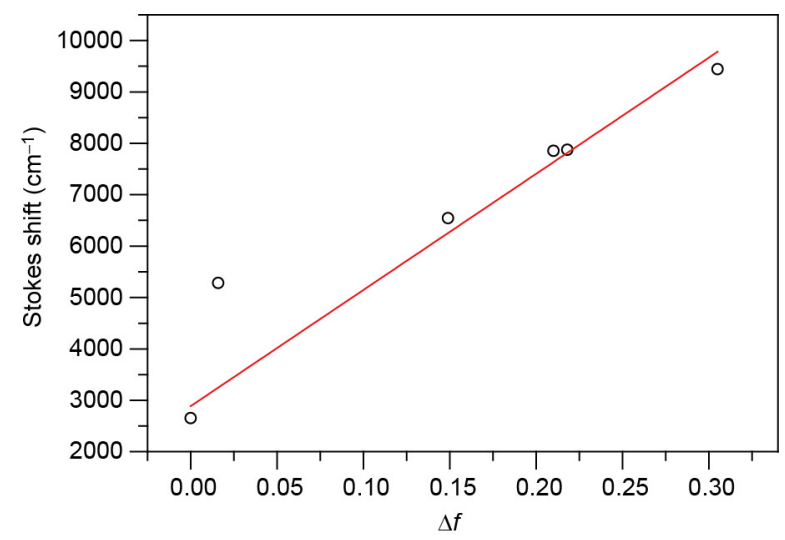

图 3 2PXZ-OXD分子的Stokes位移随溶剂极性参数的变化. 红线为拟合结果(网络版彩图)

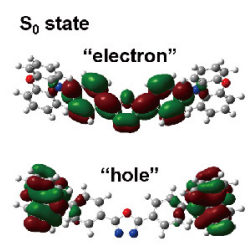

(a)

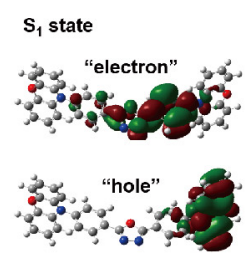

(b)

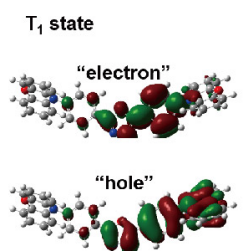

(c)
图 4 2PXZ-OXD的自然跃迁轨道分析. (a) $\mathrm{S}_{0}$; (b) $\mathrm{S}_{1}$; (c) $\mathrm{T}_{1}$ (网络版彩图)

称性. 而在 $\mathrm{S}_{1}$ 态结构基础上的分析可以看出分子对称 性打破, 形成定域在其中一个D-A部分的偶极子, 轨道 重叠部分很小, 此时具有较大的偶极, $\mathrm{S}_{1}$ 态具有较大的 永久偶极矩 $(20.4 \mathrm{D})$. 与 $\mathrm{S}_{1}$ 态相比较, $\mathrm{T}_{1}$ 态处于中心电 子受体部分具有明显的 $\pi-\pi^{*}$ 的跃迁, 轨道重叠部分明 显增加, 三重态中的局域激发态特征更加显著, 这也导 致 $\mathrm{T}_{1}$ 态的偶极矩明显减小, 仅为 $9.3 \mathrm{D}$. 通过计算获得 的激发态偶极矩, 我们认为溶剂极性对于激发态偶极 矩大的 $\mathrm{S}_{1}$ 态的影响要比对激发态偶极矩小的 $\mathrm{T}_{1}$ 态的影 响强很多. 因此, 溶剂对于 $S_{1}$ 和 $T_{1}$ 轨道能级的调控则直 接影响 2PXZ-OXD的发光及延迟苂光特性. 以下将对 其发光性质和溶剂化激发态动力学进行进一步阐释.

\section{4 苂光量子产率与寿命}

表1中同时列出了不同溶剂中2PXZ-OXD的苂光 量子产率和苂光寿命. 苂光衰减曲线如图 S1 (补充材 料)所示. 溶剂与激发态溶质分子的相互作用, 导致其 激发态能级的下降, 尤其是具有强偶极的 $\mathrm{S}_{1}$ 态. 因此, 苂光特性会受到溶剂极性的显著影响.

随着溶剂极性的增加, 分子激发态中的极性电荷 转移态的成分越来越大, 电荷转移态的轨道重叠要 明显小于局域激发态, 轨道重叠的减小导致其发光 效率变低. 特别地, 我们发现在非极性的环已烷里, 荧光量子产率非常低. 这主要是由于非极性溶剂中 2PXZ-OXD分子容易保持对称性结构, 具有明显的四 极矩分子特点, 弱极性溶剂导致其没有电荷转移的特 点. 其单重态能级较高, 同时由于系间窝越生成更多 的三重态而由于能级差比较大导致反向系间窝越的 机率小, 并且在空气饱和下, 三重态的寿命仅为 $50 \mathrm{~ns}$ 左右, 氧气对三重激发态的猝灭效应显著, 从而造成 其荧光量子产率较低. 随着极性的增加, 单重态能级 下降, 导致其三重态生成效率变低, 并且因为单重态- 
三重态能级差减小, 导致反向系间鼌越的可能性增加, 使甲苯溶液中的量子产率较高. 但是随着溶剂极性的 进一步增加, 单重态的发光振子强度减小, 非辐射跃 迁的成分增多. 由于能级调控及溶剂化猝灭, 导致量 子产率会在弱极性的甲苯中达到最大值. 在强极性的 乙腈中, 单重态能级进一步降低, 甚至低于 $\mathrm{T}_{1}$ 的能级 而不能有效发生系间暲越, 溶剂化弛豫的苂光猝灭占 据主导, 几乎没有荧光, 寿命明显减小.

延迟荧光的测量在 $\mathrm{N}_{2}$ 饱和溶液中完成. 图 $\mathrm{S} 2$ 中给 出了其在微秒区间内的衰减动力学, 由于仪器响应函 数的限制, 在此仅关注长寿命的延迟荧光的动力学过 程. 延迟荧光寿命在环己烷和四氢呋喃中均为单指数 拟合, 寿命分别为 6.3 和 $2.7 \mu \mathrm{s}$. 而在甲苯溶液中双指数 拟合分别为 $4.3 \mu \mathrm{s}(44 \%)$ 和 $17.9 \mu \mathrm{s}(56 \%)$. 在强极性的 乙腈溶液中, 即使在除氧条件下也没有延迟苂光产生, 这与前面分析的溶剂化过程是一致的. 在稳态实验及 量化计算结果中已经提及, 溶剂极性对单重激发态 $\mathrm{S}_{1}$ 的能级的调控要远大于基态 $\mathrm{S}_{0}$ 和三重激发态 $\mathrm{T}_{1}$. 因为 $\mathrm{S}_{1}$ 和 $\mathrm{T}_{1}$ 态具有显著差别的偶极矩大小, 在考虑延迟苂 光产生的机理时, 我们认为, $\mathrm{T}_{1}$ 态的能级几乎不受溶 剂影响, 因此, 单重态-三重态之间的能级差改变主要 来源于在极性溶剂中单重态能级的显著降低. 在量化 计算优化结构中, 基态与激发态结构有所不同, 尤其 是电子给体和电子受体间的二面角, 可能存在由于分 子构型改变导致的多个电荷转移态, 对于这一推测在 下一节进一步对其激发态动力学进行了探究 ${ }^{[28]}$.

\section{5 瞬态吸收光谱实验}

通过对2PXZ-OXD在不同溶剂中的飞秒瞬态吸收

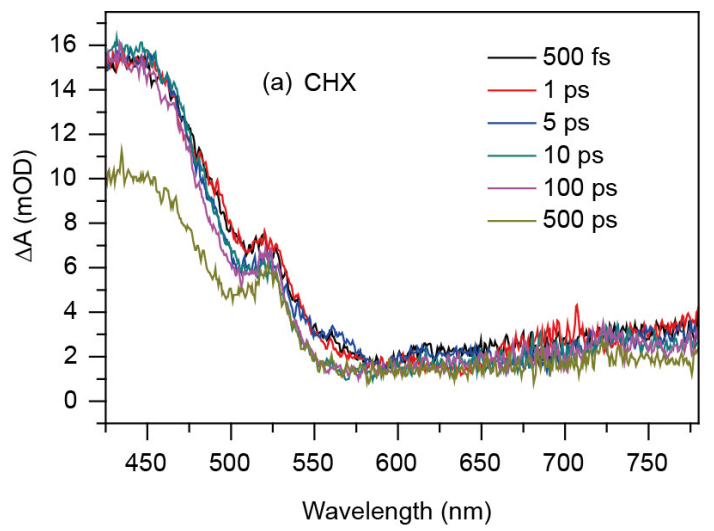

光谱实验分析来更加深入地探究分子构型变化对激发 态电荷转移的动力学过程的影响. 图5 给出了在环己 烷和甲苯溶液中的不同延迟时刻的瞬态吸收光谱, 激 发波长选择 $400 \mathrm{~nm}$. 可以看出, 这一分子体系具有 3 个 激发态吸收信号, 分别位于 $500 \mathrm{~nm}$ 之前, 500 550 nm及 大于 $600 \mathrm{~nm}$. 在两个溶液体系中, 处于 $500 \mathrm{~nm}$ 之前的 激发态吸收在 $10 \mathrm{ps}$ 时达到最大值. 而两者处于 $520 \mathrm{~nm}$ 的信号有所不同: 在环己烷中与 $500 \mathrm{~nm}$ 前的信号同时 衰减, 在甲苯中却有一个慢的生成过程. 为了进一步 理解激发态动力学过程, 使用全局拟合的方法处理瞬 态吸收原始数据.

如图6所示为全局拟合的结果. 非极性的环己烷 溶液中2PXZ-OXD的激发态中电荷转移态成分相对单 一, 因此选用 $\mathrm{S}_{1}^{*} \rightarrow \mathrm{S}_{1} \rightarrow \mathrm{S}_{0}$ 模型, 并伴随着 $\mathrm{S}_{1}^{*}\left(\right.$ 或 $\mathrm{S}_{1}$ ) 向 $\mathrm{T}_{1}$ 态的系间鼌越过程. 在此将三重态寿命固定为无限 长, 从而拟合得到 $S_{1}^{*}$ 和 $\mathrm{S}_{1}$ 的寿命分别为 $5.8 \mathrm{ps}$ 和 $1.2 \mathrm{~ns}$. $\mathrm{S}_{1}$ 的寿命与苂光寿命一致, 而 $\mathrm{S}_{1}^{*}$ 的寿命则归属为激发 态结构弛豫过程.

由于溶剂极性的增加, 导致激发态中电荷转移成 分的不断增加. 鉴于溶液中分子结构的改变, 我们将 电荷转移态分为 $\mathrm{CT}$ 和 $\mathrm{CT}^{\prime}$ 态. 如图6(b) 所示, 甲苯中的 模型则使用 $\mathrm{S}_{1}^{*} \rightarrow \mathrm{CT} \rightarrow \mathrm{CT}^{\prime} \rightarrow \mathrm{S}_{0}$, 由于 $\mathrm{CT}^{\prime}$ 能级的下 降, 同时伴随从 $\mathrm{S}_{1}^{*}$ 和 $C \mathrm{~T}$ 态向 $\mathrm{T}_{1}$ 态的系间窝越过程. 利 用这一模型, 我们拟合出 $\mathrm{S}_{1}^{*} 、 \mathrm{CT}$ 和 $\mathrm{CT}^{\prime}$ 的寿命分别为 $3.3 \mathrm{ps} 、 75 \mathrm{ps}$ 和 $3 \mathrm{~ns}$. $\mathrm{CT}^{\prime}$ 态的寿命与荧光寿命一致, $\mathrm{S}_{1}^{*} \rightarrow \mathrm{CT}$ 的过程则是伴随溶剂化过程, 而 $\mathrm{CT} \rightarrow \mathrm{CT}^{\prime}$ 则 是结构弛豫过程. 利用同一模型对四氢呋喃溶液中拟 合, 寿命依次为 $1.9 \mathrm{ps} 、 100 \mathrm{ps}$ 和 $3 \mathrm{~ns}$. 详细拟合结果 与其动力学拟合见补充材料.

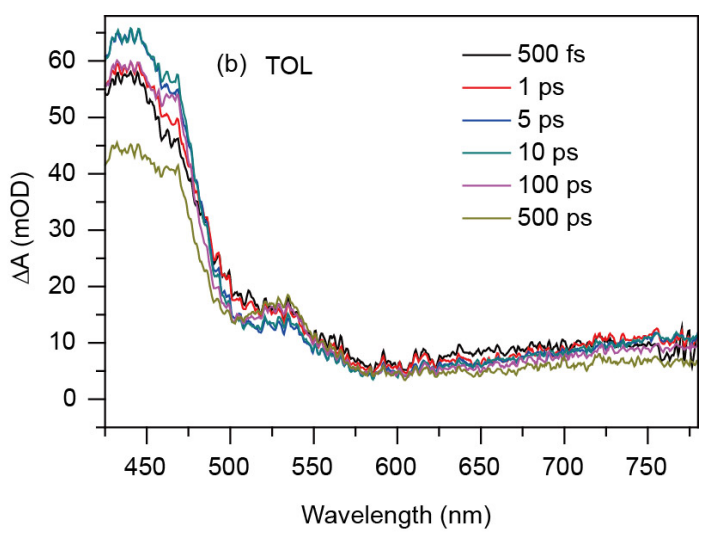

图 5 2PXZ-OXD的飞秒瞬态吸收光谱. (a) 环己烷; (b) 甲苯(网络版彩图) 

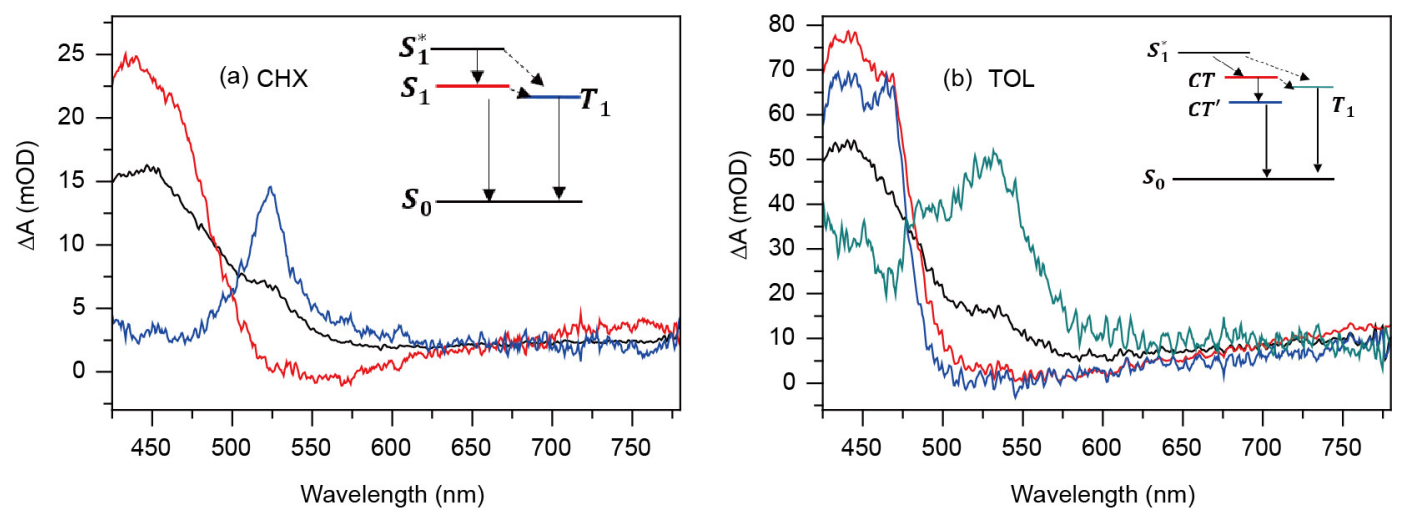

图 6 分别利用环己烷(a)和甲苯(b)模型进行目标全局拟合的组分相关光谱(网络版彩图)

值得注意的是, 在不同的溶剂中拟合出的 $\mathrm{T}_{1}$ 态的 光谱具有一定相似性, 为了进一步验证三重态存在的 这一特点, 我们利用纳秒瞬态吸收方法对这一体系进 行表征. 由于在空气中, 2PXZ-OXD的三重态寿命很短 约为 $50 \mathrm{~ns}$, 采用 $\mathrm{N}_{2}$ 饱和的溶液体系进行实验. 实验采 用激发波长为 $355 \mathrm{~nm}$. 图 7 是 $\mathrm{N}_{2}$ 饱和下环己烷及甲苯 溶液中的纳秒瞬态吸收光谱, 可以看出有 3 个瞬态吸 收信号, 分别在 $400 、 520$ 和 $600 \mathrm{~nm}$ 以上. 在文中列出 了环己烷及甲苯的光谱图, 其他数据见补充材料. 实 验所得光谱与在飞秒瞬态吸收的全局拟合中得到的 三重态信号相符合, 不同溶剂环境中2PXZ-OXD的三 重态寿命分别为 $8.4 \mu \mathrm{s}$ (环己烷)、13.5 $\mu \mathrm{s}$ (甲苯)和 2.6 $\mu \mathrm{s}$ (四氢呋喃). 从实验分析数据中, 可以得出由于乙腈 的强极性溶剂化猝灭效应, 系间窝越(S-T)过程几乎没 有来得及发生, 在 $\mathrm{N}_{2}$ 饱和条件下, 也没有三重态信号.

\section{6 激发态驰豫机理}

如量化计算中所得到的信息, 电荷转移型分子具

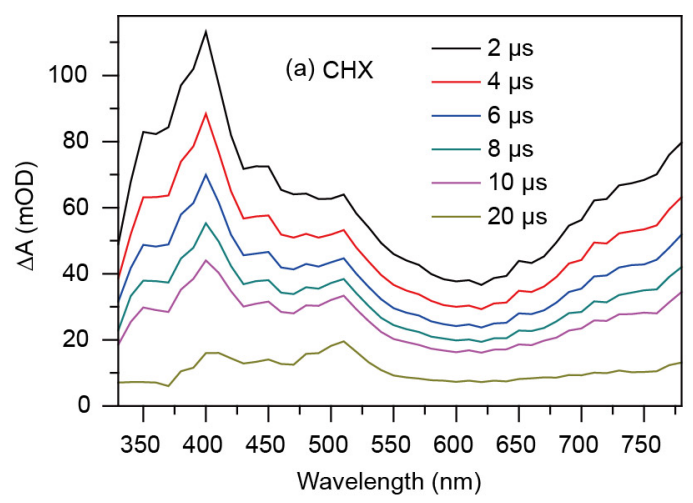

有的强偶极的单重激发态, 会受到溶剂环境的调控. 而其三重激发态具有一定的局域激发特性, 受溶剂调 控相对较弱, 通常认为其在不同极性中处于恒定值. 因此, 溶剂可以有效调整单重态-三重态能级差, 从而 影响电荷转移型分子的延迟苂光特性.

在2PXZ-OXD这一体系中, 从非极性到强极性溶 剂, 苂光光谱的明显红移证明了发光态能量随着极性 增加而不断降低. 非极性溶剂中2PXZ-OXD分子容易 保持对称性结构, 具有明显的四极矩分子特征, 从而 导致弱极性溶剂对其结构影响甚微而难以形成电荷 转移的动力学过程. 而随着极性的增加, 分子对称性 容易打破, 形成强偶极的电荷转移态, 定域在其中某 一个D-A侧枝上. 由于分子中电子给受体能力强, 即使 弱极性的甲苯也能诱导对称性打破形成强极性的电 荷转移态. 由于四极分子结构弛豫的存在, 造成多个 CT态的存在 ${ }^{[29]}$.

在环已烷中, 由于单重激发态能级较高, 可以直

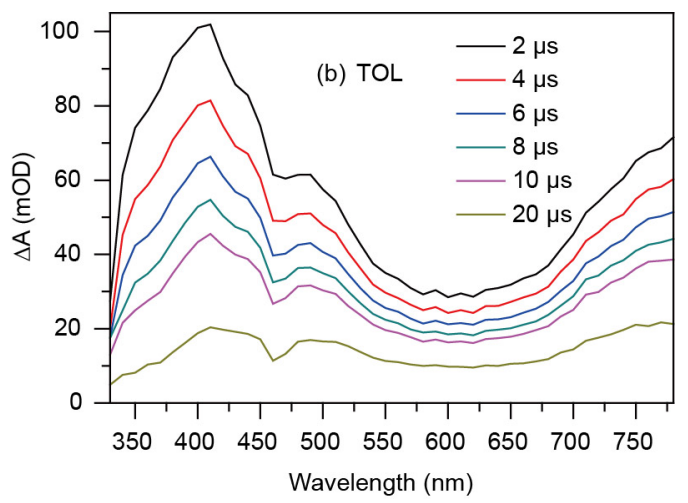

图 7 2PXZ-OXD在环已烷(a)和甲苯(b)中的纳秒瞬态吸收光谱(网络版彩图) 
接通过系间窝越过程到达三重态, 而由于两能级之间 的能隙相对较大, 从而导致其反向系间窝越回单重态 的机率变小. 而随着溶剂极性的增加, 导致对称性打 破的电荷转移态的能级下降, 使其与三重态之间的能 级差变小. 在甲苯中, 三重态能级可能存在于 $\mathrm{CT}$ 与 $\mathrm{CT}^{\prime}$ 态之间, 从而造成了延迟荧光的两个寿命. 而极性更 大的四氢呋喃中, 溶剂化导致单重态能级继续下降, 从而使 CT 态可能处于三重态能级之下, 形成更快的反 向系间鼌越. 在强极性的乙腈中, 由于溶剂化诱导的 电荷转移态快速的内转换无辐射弛豫过程, 完全抑制 了系间窝越过程, 没有观测到三重态信号.

\section{4 结论}

通过稳态、瞬态光谱测量及量化计算, D-A-D型 分子 2 PXZ-OXD的激发态动力学得以深入探究. 稳态 光谱及溶剂化结构表明这一分子具有明显的电荷转 移特性, 这一特点是热活化延迟苂光材料的必备条件. 瞬态吸收光谱及发光动力学对该分子的激发态动力学 过程的研究结果进一步表明, 溶剂化诱导单重态能级 的下降减小了单重态-三重态能级差, 有利于延迟苂光 的增强, 但同时溶剂极性会增强非辐射弛豫, 导致延迟 荧光的减弱. 这两个方面的相互制衡是利用延迟苂光 分子制备有机功能器件过程中需要考量的基本问题.

\section{补充材料}

本文的补充材料见网络版http://chemen.scichina.com. 补充材料为作者提供的原始数据, 作者对其学术质量和内容负责.

\section{参考文献}

Tang CW, VanSlyke SA. Appl Phys Lett, 1987, 51: 913-915

2 Baldo MA, Thompson ME, Forrest SR. Pure Appl Chem, 1999, 71: 2095

3 Baldo MA, O’Brien DF, You Y, Shoustikov A, Sibley S, Thompson ME, Forrest SR. Nature, 1998, 395: 151-154

4 Tao Y, Yuan K, Chen T, Xu P, Li H, Chen R, Zheng C, Zhang L, Huang W. Adv Mater, 2014, 26: 7931-7958

5 Wang H, Xie L, Peng Q, Meng L, Wang Y, Yi Y, Wang P. Adv Mater, 2014, 26: 5198-5204

6 Méhes G, Nomura H, Zhang Q, Nakagawa T, Adachi C. Angew Chem Int Ed, 2012, 51: 11311-11315

7 Madigan CF, Bulović V. Phys Rev Lett, 2003, 91: 247403

8 Bulović V, Deshpande R, Thompson ME, Forrest SR. Chem Phys Lett, 1999, 308: 317-322

9 Hu J, Li Y, Zhu H, Qiu S, He G, Zhu X, Xia A. ChemPhysChem, 2015, 16: 2357-2365

10 He G, Shao J, Li Y, Hu J, Zhu H, Wang X, Guo Q, Chi C, Xia A. Phys Chem Chem Phys, 2016, 18: 6789-6798

11 Zhu H, Li M, Hu J, Wang X, Jie J, Guo Q, Chen C, Xia A. Sci Rep, 2016, 6: 24313

12 Fan D, Yi Y, Li Z, Liu W, Peng Q, Shuai Z. J Phys Chem A, 2015, 119: 5233-5240

13 Chen XK, Zhang SF, Fan JX, Ren AM. J Phys Chem C, 2015, 119: 9728-9733

14 Lee J, Shizu K, Tanaka H, Nomura H, Yasuda T, Adachi C. J Mater Chem C, 2013, 1: 4599

15 Zhao Y, Truhlar DG. Acc Chem Res, 2008, 41: 157-167

16 Martin RL. J Chem Phys, 2003, 118: 4775-4777

17 Frisch MJ, Trucks GW, Schlegel HB, Scuseria GE, Robb MA, Cheeseman JR, Scalmani G, Barone V, Mennucci B, Petersson GA, Nakatsuji H, Caricato M, Li X, Hratchian HP, Izmaylov AF, Bloino J, Zheng G, Sonnenberg JL, Hada M, Ehara M, Toyota K, Fukuda R, Hasegawa J, Ishida M, Nakajima T, Honda Y, Kitao O, Nakai H, Vreven T, Montgomery JA Jr., Peralta JE, Ogliaro F, Bearpark M, Heyd JJ, Brothers E, Kudin KN, Staroverov VN, Keith T, Kobayashi R, Normand J, Raghavachari K, Rendell A, Burant JC, Iyengar SS, Tomasi J, Cossi M, Rega N, Millam JM, Klene M, Knox JE, Cross JB, Bakken V, Adamo C, Jaramillo J, Gomperts R, Stratmann RE, Yazyev O, Austin AJ, Cammi R, Pomelli C, Ochterski JW, Martin RL, Morokuma K, Zakrzewski VG, Voth GA, Salvador P, Dannenberg JJ, Dapprich S, Daniels AD, Farkas O, Foresman JB, Ortiz JV, Cioslowski J, Fox DJ. Gaussian 09 (Revision D. 01), 2013, Gaussian, Inc., Wallingford 


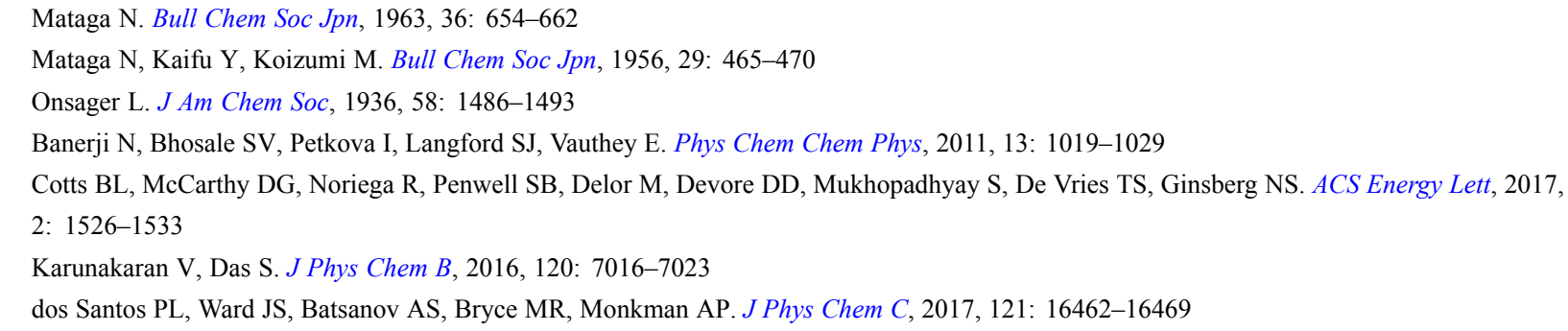

\title{
Solvent polarity dependence on excited charge-transfer state properties in donor-acceptor-donor thermally activated delayed-fluorescence molecule
}

\author{
Guiying $\mathrm{He}^{1,2}$, Zhuoran Kuang ${ }^{1,2}$, Xian Wang ${ }^{1,2}$, Hongwei Song ${ }^{1,2}$, Qianjin Guo ${ }^{1 *}$, Andong Xia ${ }^{1,2^{*}}$ \\ ${ }^{1}$ Beijing National Laboratory for Molecular Sciences (BNLMS) and Key Laboratory of Photochemistry, Institute of Chemistry, Chinese Academy of \\ Sciences, Beijing 100190, China \\ ${ }^{2}$ University of Chinese Academy of Sciences, Beijing 100049, China \\ *Correspondingauthors (email: guoqj@iccas.ac.cn; andong@iccas.ac.cn)
}

\begin{abstract}
The photophysical properties of a donor-acceptor-donor (D-A-D) molecule based on oxadiazole, 2,5-bis(4-(10H-phenoxazin-10-yl)phenyl-1,3,4-oxadiazole (2PXZ-OXD), have been systematically investigated by means of steady state and transient spectroscopy. A significant increase of Stokes shift with increasing solvent polarity indicates that a dipolar charge-transfer state is produced upon excitation. Further quantum chemical calculations show that the dipole moment of singlet excited state is much larger than that of triplet excited state, where singlet excited state is much more dependent on the polarity of solvents. The symmetry-breaking charge transfer states of quadrupole molecule induced by solvent polarity, exhibit large energy loss of singlet state with increasing of polarity. The energy gap is controlled by solvent polarity because of solvation. Furthermore, the emissive oscillator strength is dependent on the polarity, and the non-emissive relaxation resulted from the strong solvation reduces the luminescence properties of emitter.
\end{abstract}

Keywords: charge transfer, thermally activated delayed fluorescence (TADF), solvation doi: $10.1360 / \mathrm{N} 032017-00149$ 